Supporting Information

\title{
Non-ribosomal Peptide Extension by a Peptide Amino-acyl tRNA Ligase
}

\author{
Zhengan Zhang, ${ }^{1}$ and Wilfred A. van der Donk ${ }^{1,2 *}$
}

\begin{abstract}
Affiliations:
${ }^{1}$ Department of Chemistry, University of Illinois at Urbana-Champaign, Urbana, IL, USA. ${ }^{2}$ Howard Hughes Medical Institute, University of Illinois at Urbana-Champaign, Urbana, IL, USA.

* Corresponding author:

Wilfred A. van der Donk (vddonk@illinois.edu), phone: 1-217-244-5360, fax: 1-217-244-8533
\end{abstract}

\section{Table of Contents:}

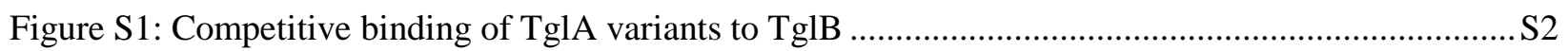

Figure S2: TglB is relatively promiscuous towards a change of the last amino acid of TglA ...................S4

Figure S3: $P$. syringae tRNA ${ }^{\mathrm{Cys}}$ mini- or micro-helix is sufficient to catalyze the in vitro cysteinylation

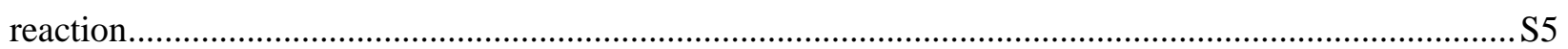

Figure S4: TglB hydrolyzes ATP in the absence of substrate TglA................................................... 6

Figure S5: Potential ping-pong mechanism involving the formation of an aminoacyl-enzyme intermediate

Figure S6: Sequence alignment of TglB homologs

Figure S7: Mutagenesis of selective Ser, Thr and Tyr residues of TglB does not support the ping-pong mechanism in Figure S4 or Figure S7.

Figure S8: Potential mechanism in which TglA is covalently linked to a hydroxyl group of TglB

Figure S9: A potential mechanism in which the C-terminus TglA is first activated by ATP, followed by attack by a carboxylate residue on TglB to form an anhydride S14

Figure S10: A potential ping-pong mechanism with a covalent acyl-phosphate intermediate S15

Figure S11: SDS-PAGE analysis of TglB variants with diminished activity S16

Figure S12: MALDI-TOF-MS spectra of in vitro cysteinylation of TglA with TglB variants S17

Figure S13: MALDI-TOF-MS spectra of $\mathrm{NH}_{2} \mathrm{OH}$ quenching assays with TglB dead mutants S18

Table S1: Activity of alanine-substituted TglB proteins S19

Table S2: Oligonucleotide primers used in this study S20 
Figure S1. Competitive binding of TglA variants to TglB. Competitive fluorescence polarization traces for binding of wild-type (WT) TglA and TglA variants to TglB. These peptides were used to titrate TglB $(3 \mu \mathrm{M})$ complexed with fluorescein-labeled peptide consisting of the 20 C-terminal amino acids of TglA $(25 \mathrm{nM})$. Error bars represent standard deviation (s.d.) of three independent replicates. Errors on $\mathrm{IC}_{50}$ are the standard error of the mean (s.e.m.) given by regression analysis.
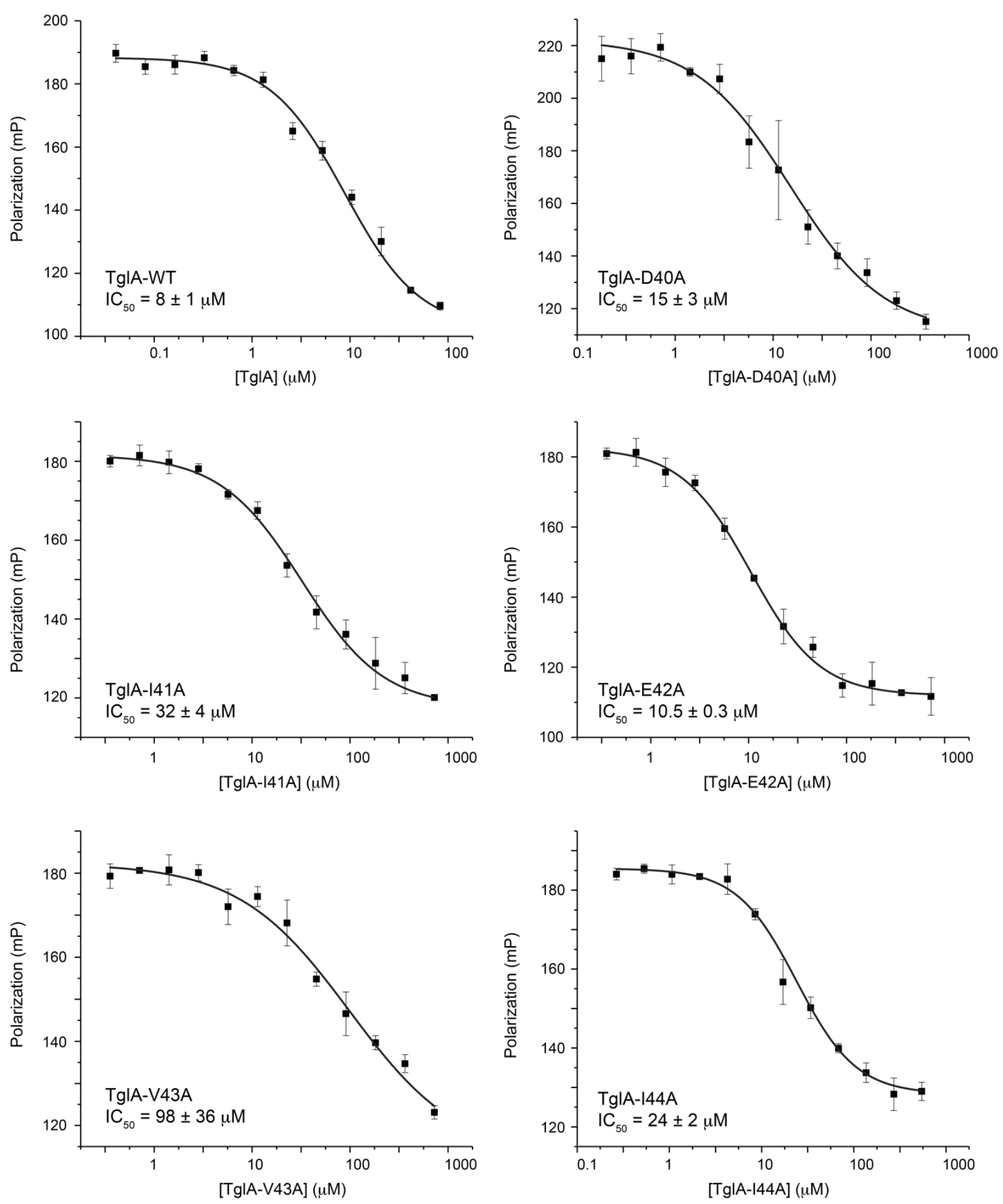

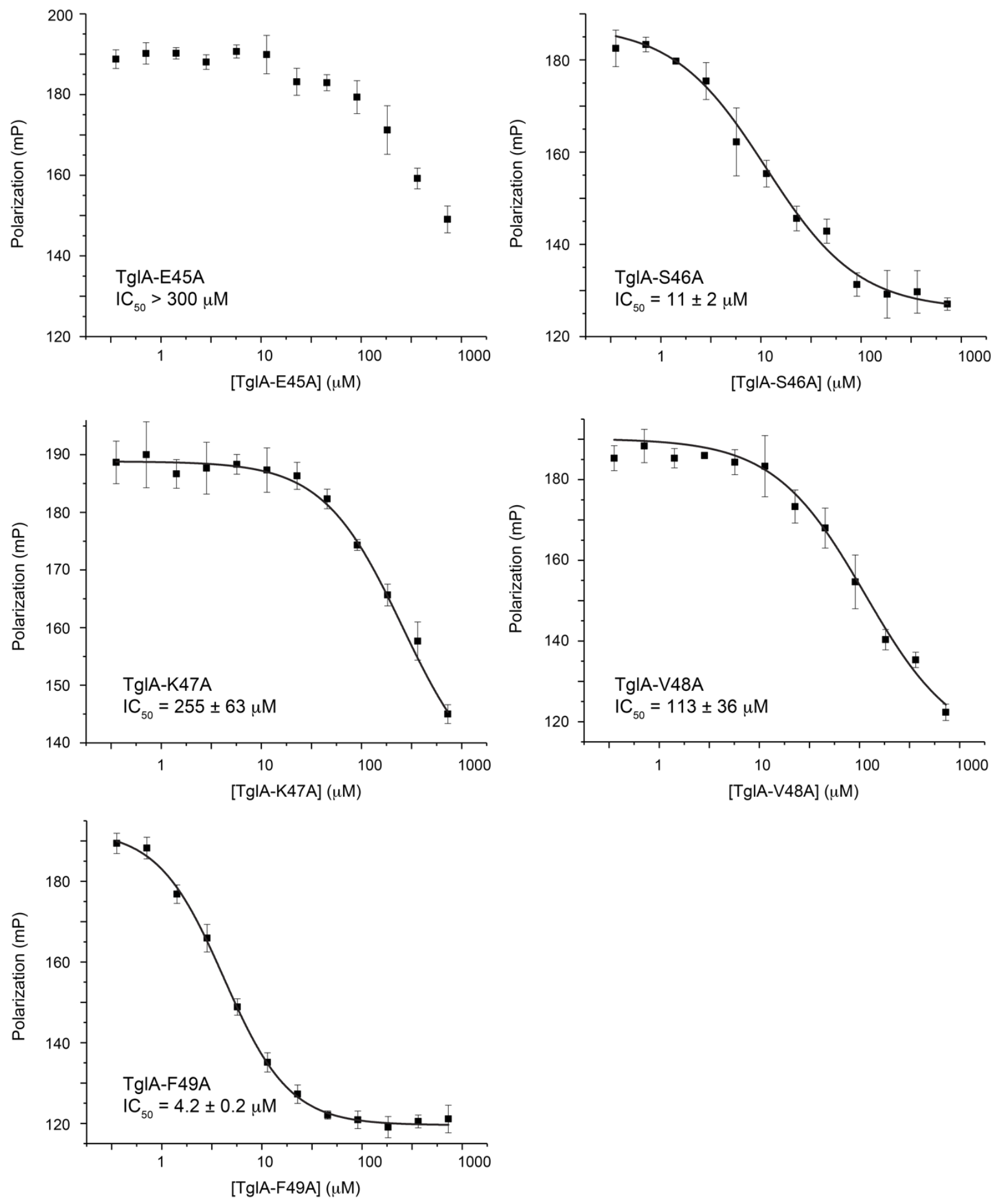

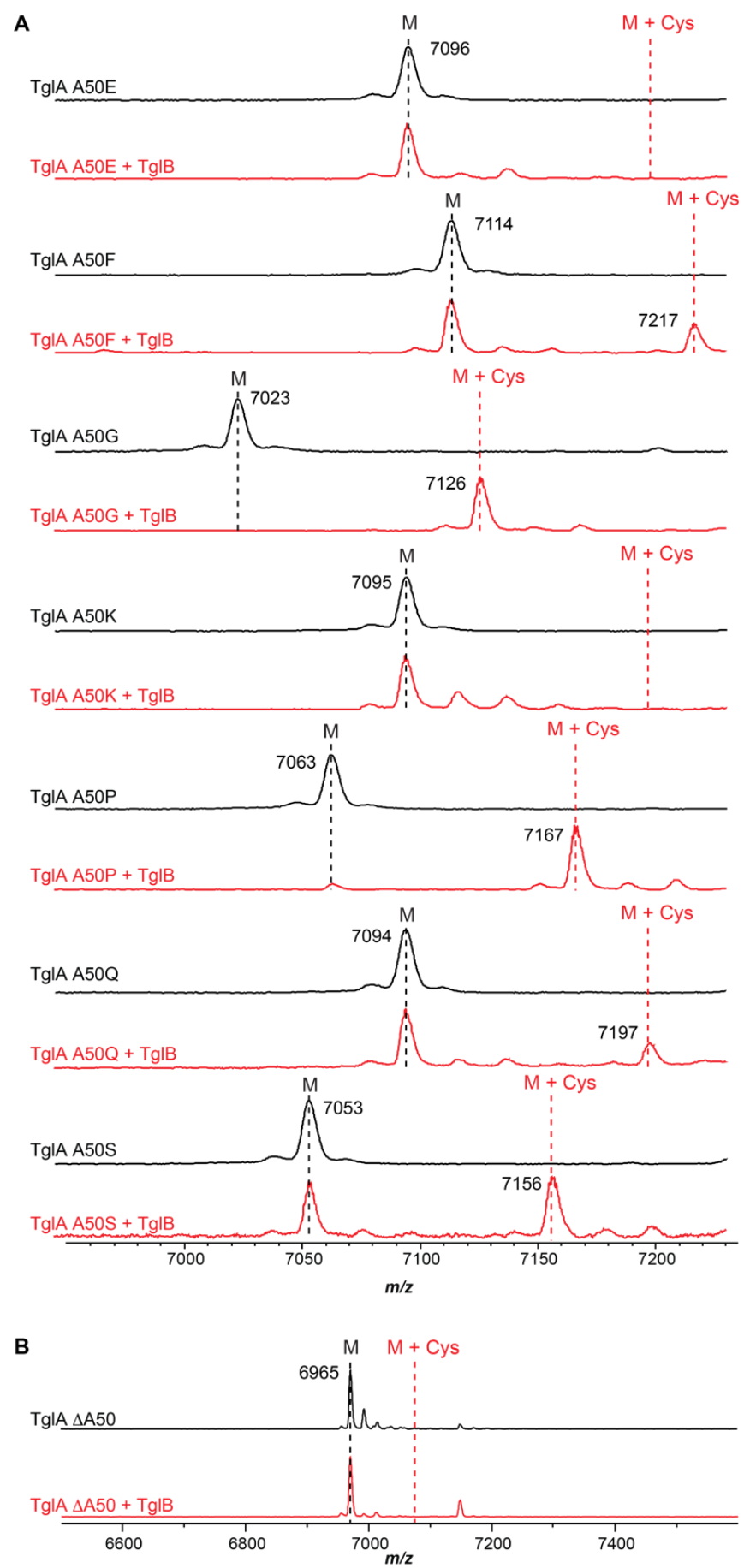

Figure S2. TglB is relatively promiscuous towards a change of the last amino acid of TglA. MALDITOF mass spectra of (A) TglA-Ala50 variants and their co-expression products with TglB in E. coli. (TglA A50E, calc. $[\mathrm{M}+\mathrm{H}]^{+}, 7095$. TglA_A50F, calc. $[\mathrm{M}+\mathrm{H}]^{+}, 7114$; TglA_A50F_Cys, calc. $[\mathrm{M}+\mathrm{H}]^{+}, 7217$. TglA_A50G, calc. $[\mathrm{M}+\mathrm{H}]^{+}, 7023$; TglA_A50G_Cys, calc. $[\mathrm{M}+\mathrm{H}]^{+}, \overline{7}$ 7127. TglA_A50K, calc. $[\mathrm{M}+\mathrm{H}]^{+}$, 7095. TglA_A50P, calc. $[\mathrm{M}+\mathrm{H}]^{+}$7063; TglA_A50P_Cys, calc. $[\mathrm{M}+\mathrm{H}]^{+}, 7167$. TglA_A50Q, calc. $[\mathrm{M}+\mathrm{H}]^{+}$, 7094; TglA_A50Q_Cys, calc. [M+H] $]^{+}, 7198$. TglA_A50S, calc. [M+H] $]^{+}, 7053$; TglA_A50S_Cys, calc. $[\mathrm{M}+\mathrm{H}]^{+}$, 7157). (B) TglA $\triangle \mathrm{A} 50$ variant and its product after co-expression with TglB in E. coli. (TglA_ $\Delta$ A50, calc. $\left.[\mathrm{M}+\mathrm{H}]^{+}, 6966\right)$. All $\mathrm{m} / \mathrm{z}$ values are average masses. 
A

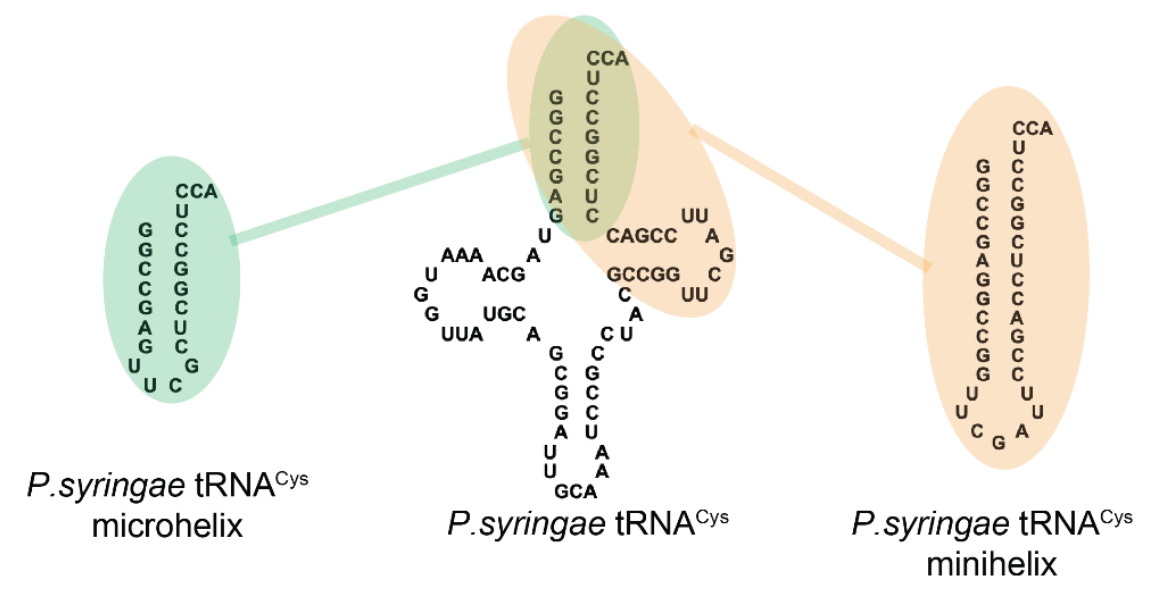

B

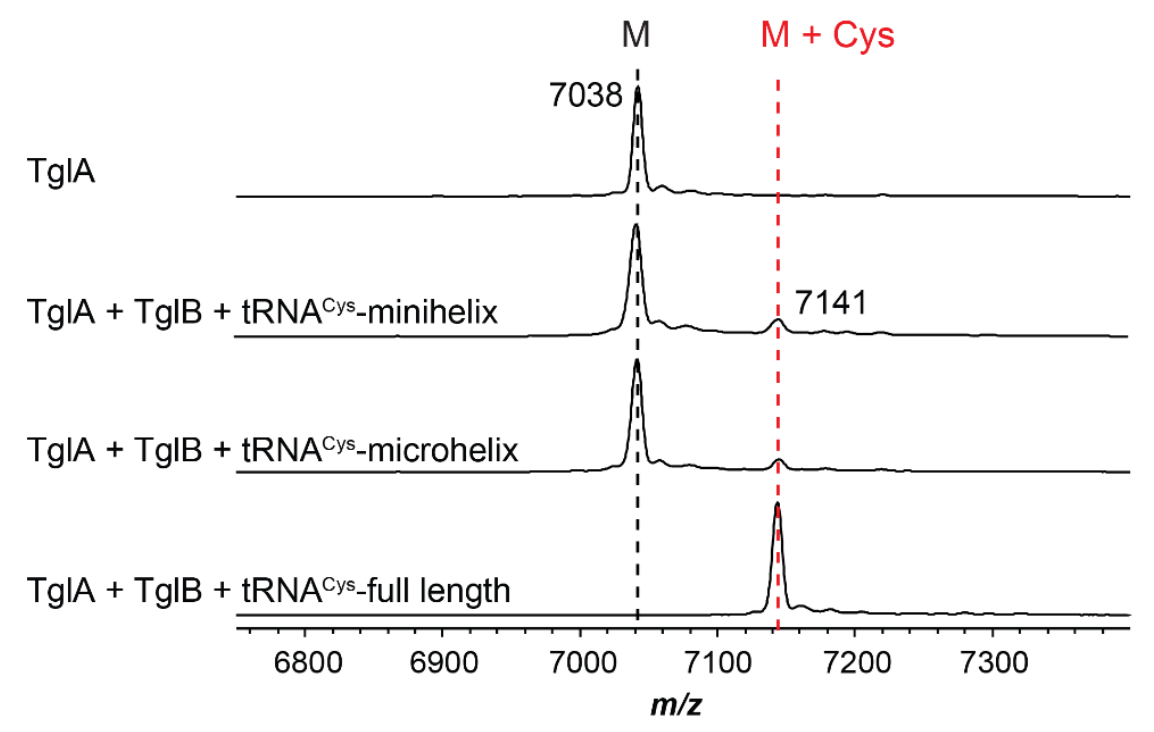

Figure S3. $P$. syringae tRNA ${ }^{\mathrm{Cys}}$ mini- or micro-helix is sufficient to catalyze the in vitro cysteinylation reaction. (A) Secondary structure of full length $P$. syringae tRNA $^{\text {Cys }}$, and its mini- or micro-helix. (B) MALDI-TOF-MS analysis of in vitro cysteinylation reactions with $P$. syringae tRNA ${ }^{\text {Cys }}$ mini- or microhelix under standard assay conditions. (TglA, calc. $[\mathrm{M}+\mathrm{H}]^{+}, 7037$; TglA_Cys, calc. $[\mathrm{M}+\mathrm{H}]^{+}, 7141$ ). 


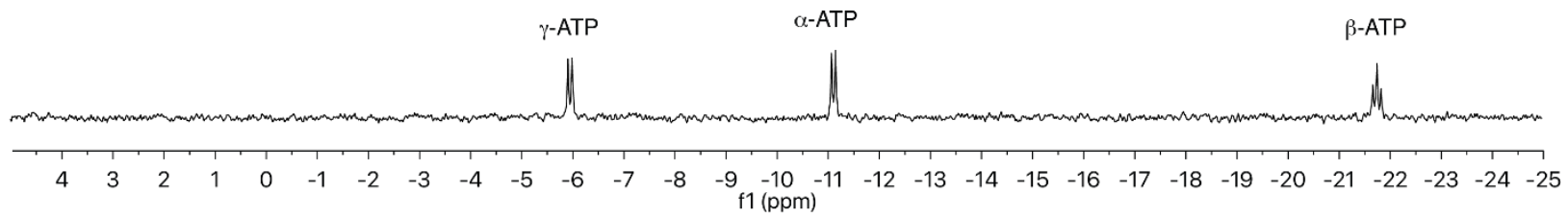

B

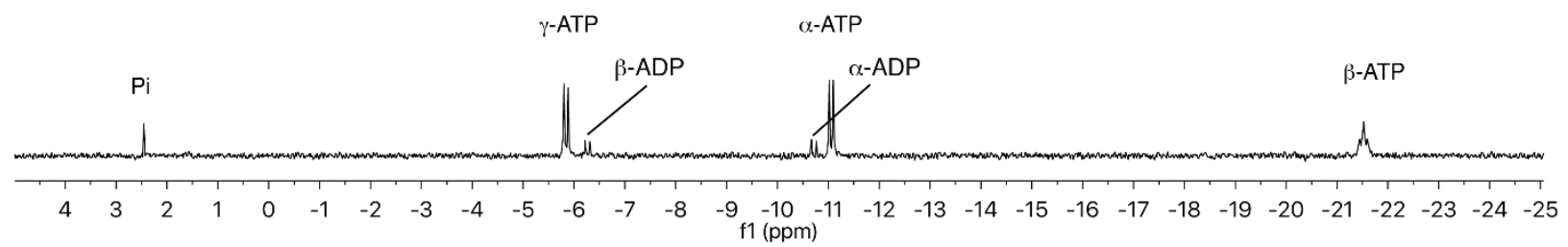

C

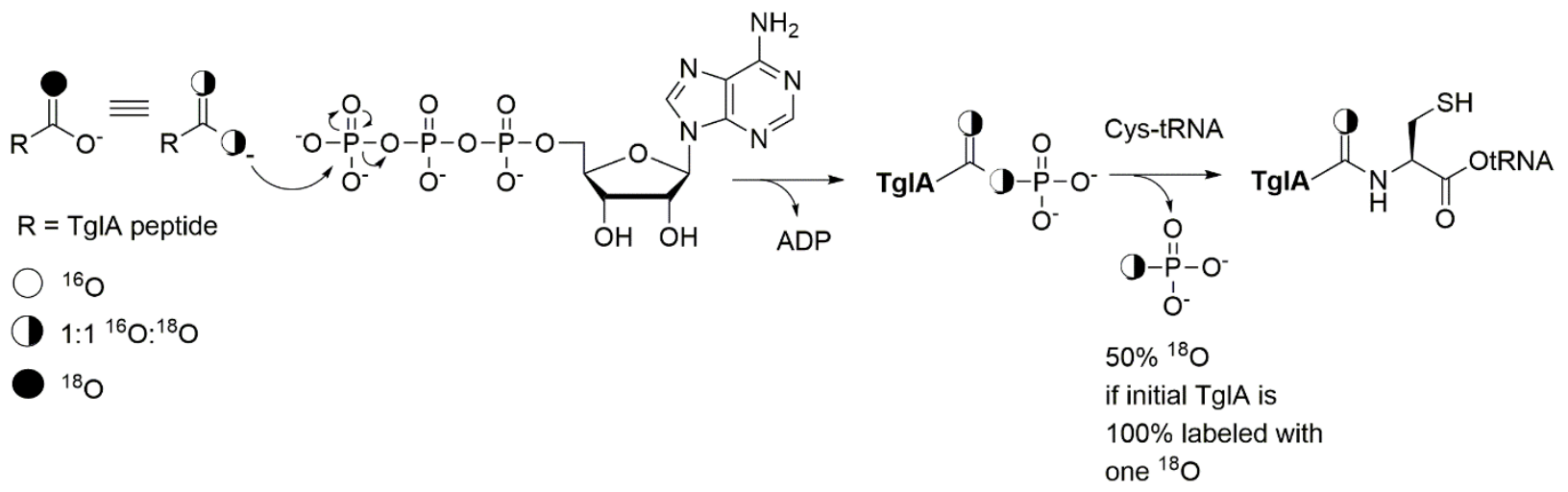

Figure S4. TglB hydrolyzes ATP in the absence of substrate TglA. ${ }^{31} \mathrm{P}$ NMR analysis of $500 \mu \mathrm{M}$ ATP incubated in HEPES assay buffer (100 mM HEPES pH 7.5; $\left.5 \mathrm{mM} \mathrm{MgCl}_{2}, 100 \mathrm{mM} \mathrm{NaCl}\right)$ at $30^{\circ} \mathrm{C}$ for 30 min in the absence (A) and presence of $50 \mu \mathrm{M} \mathrm{TglB} \mathrm{(B).} \mathrm{(C)} \mathrm{If} \mathrm{TglA} \mathrm{was} 100 \%$ labeled with one ${ }^{18} \mathrm{O}$ at the C-terminal carboxylate, then upon phosphorylation $50 \%$ of the label would be in the carbonyl group and $50 \%$ in the bridging oxygen of the phosphate ester. Upon attack by the Cys-tRNA, this would lead to 50\% of labeled phosphate. Using the same reasoning, when $85 \%$ of TglA is labeled with one ${ }^{18} \mathrm{O}$, then the phosphorylated peptide will be $15 \%$ unlabeled, $42.5 \%$ labeled in the carbonyl oxygen, and $42.5 \%$ in the bridging oxygen, and hence after attack by Cys-tRNA $\sim 42 \%$ of the released phosphate would be ${ }^{18} \mathrm{O}$ labeled, as mentioned in the main text. $42.5 \%{ }^{18} \mathrm{O}$ and $57.5 \%{ }^{16} \mathrm{O}$ corresponds to a calculated ratio of 1:1.35. The observed ratio was $1: 2{ }^{18} \mathrm{O}:{ }^{16} \mathrm{O}$. However, a significant amount of ${ }^{16} \mathrm{O}$ arises from hydrolysis of ADP to phosphate and AMP as shown by NMR analysis (Figure 3). The fraction of the total phosphate that was derived from ADP hydrolysis was estimated by taking the difference in integration of the phosphate peak and either of the two ADP peaks (it was harder to integrate the AMP peak which was overlapping with other peaks). This amount of phosphate produced from ADP hydrolysis was subtracted from the total amount of ${ }^{16} \mathrm{O}$-phosphate observed to arrive at the amount of ${ }^{16} \mathrm{O}$-phosphate produced from ATP. This correction leads to a ratio of ${ }^{18} \mathrm{O}:{ }^{16} \mathrm{O}$ of $1: 1.4$. 


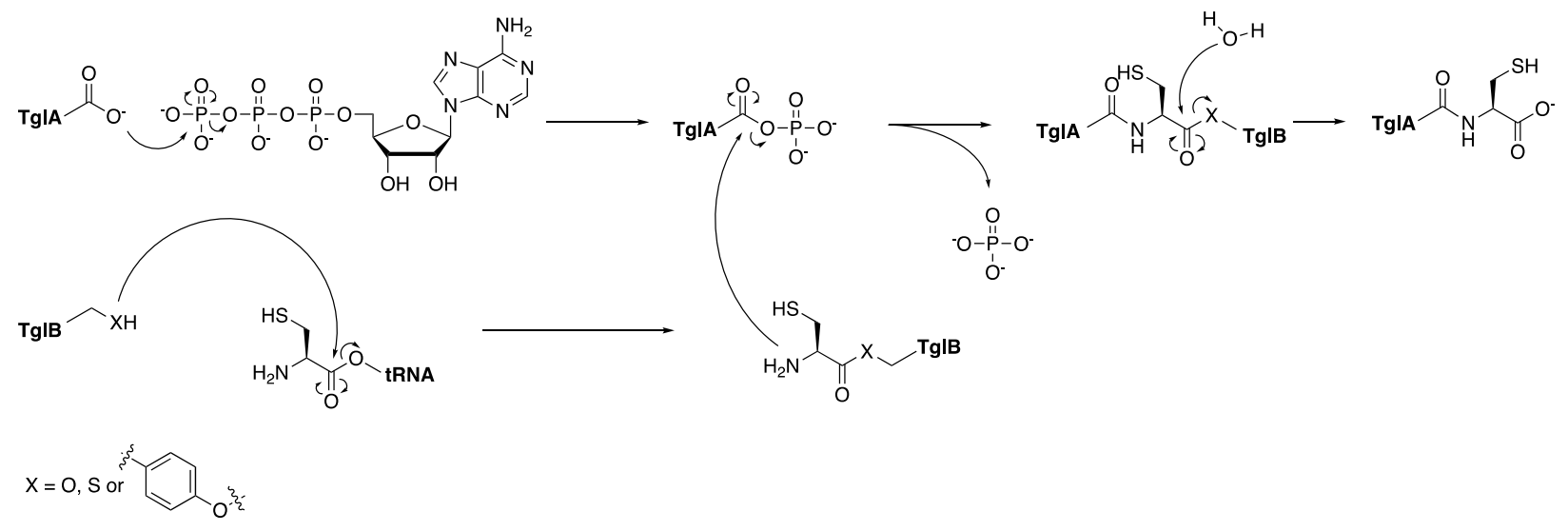

Figure S5. Potential ping-pong mechanism involving the formation of an aminoacyl-enzyme intermediate. The amino acylated tRNA first transfers the amino acid onto a residue of TglB. Then, the amino group of the tethered amino acid attacks the activated TglA peptide. Finally, hydrolysis of the protein-peptide complex releases the product. 
WP 010898205.1 $\mathrm{BA} \overline{\mathrm{B}} 05753.1$

WP 102649326.1 WP 110653318.1 $W^{-} 048368865.1$ $\mathrm{Tg} \overline{\mathrm{I}} \mathrm{B}$

WP 117166554.1 WP 112718210.1 WP 095098125.1 CŪ̄̄02180.1

WP 010898205.1 $\mathrm{BA} \overline{\mathrm{B}} 05753.1$ WP 102649326.1 WP 110653318.1 WP 048368865.1 Tg $\bar{l} \mathrm{~B}$

WP 117166554.1 WP 112718210.1 WP 095098125.1 CŪ̄o2180.1

WP 010898205.1 $\mathrm{BA} \overline{\mathrm{B}} 05753.1$ WP 102649326.1 WP_110653318.1 WP 048368865.1 $\mathrm{Tg} \overline{\mathrm{IB}}$

WP 117166554.1 $W^{-} 112718210.1$ WP 095098125.1 CUW̄02180.1

WP 010898205.1 $\mathrm{BA} \overline{\mathrm{B}} 05753.1$

WP 102649326.1 WP $^{-} 110653318.1$ WP $^{-} 048368865.1$ $\mathrm{Tg} \overline{\mathrm{IB}}$

WP 117166554.1 $\mathrm{WP}^{-} 112718210.1$ WP_095098125.1 CUW̄02180.1

WP 010898205.1 $\mathrm{BA} \overline{\mathrm{B}} 05753.1$ WP 102649326.1 WP 110653318.1 WP 048368865.1 $\mathrm{Tg} \overline{\mathrm{I}} \mathrm{B}$

WP 117166554.1 WP_112718210.1 WP 095098125.1 CŪ̄o2180.1
MI ISISNLSPKSNRSQTKDSQISKWSYFSDCILRSTGFPAEWIEKLCFHKTTTMFDLCYR --------------MWFNT S TWRWLKP FVLRS TS I P I EMVLNLRLKES I KQLNEWSK ----------------MSLSSFFW-----MRSAGFP I SWLERFAVRLDEAQVARLHA ----------------MES SQYFW-----LRS TGF P I HHLTDLGRFADL PACRVFEQ ---------------MES SQYFW---- LRS TGF P I HYLTDLGRLSELPACRAFEQ ----------------MES S HYFW-----LRSTGFAVHHLTRLGKMAELPLLKDFET $----------------M E S$ S LYFW-----LRS T GFP I HHLTQLGKMDELPLLKDFED

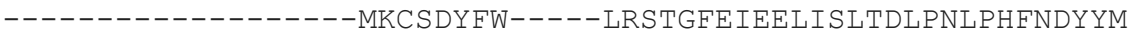
----------------MRCADYFW-----LRSAGFPADQLMQATS FPS LPAFTSLM$---------------M K C A D Y F W----$ LRSAGF PADHLMQVS PFSNLPAFTLLME $: \quad: \quad:{ }^{*}:$ : : :

TEQS LNELVERLKTLEQTCHIPSNARQDWQRIRKLVGKRKKVTDEF I HLMEQKGFDQSLT SQQS FDQWCNHWIKEIRSVPVERISLPHQRKVIRRIKKRNPLTEQDKI IVTKWGMEKLID LTES IEARIARIREGLLK-----AGS PVALKVVRKLTEGMPLTAKDWPADQTALAAAELE DFRSLQTLKATLLEKAKA-----HSPQACRKF IRKLNENQALQPTDLPEVLREPLGEALQ DFRAFQALKAMMLEEANS-----HSLLTCRKLIRKLNEGQSLLLSDLPQALREPLNTAVL DYRS LNTLRDSLLEKS IS-----HSSQACRKLIRKLNENL PLQVSDLPEALRDDAVDELA SYRCVAVLRSKLLQKSMS-----HSAQASRKLIRKLNENL PLQVSDLPEALRESSREDLE LVGQAQTIKANLKTQLIE-----FGEQSSRNF LRKLNDGEQLS IRVLPVELRGKLGDATE ---ALHQLRGRLLQQFEQ-QMAVVGEVQCRKFARKLAAQQAVSVSDLPLPLRDVLQQPLD LYQ----WRGRLLQTFEQ-QMA IVGEEQCRKFARKLAAQQAVSVSDLPLPLRDVLHQPLG . . : :

QEIESYERKRRLQEKNWTKARESFEAELAEKQLFLQEIYKNPRLQEAIFQQSPSMYKNAV ---EYCSQQQS LISLK-KKAQQSFHEEFERLQQQL IERFQSSQYELALYTLNHKLWHFWI ---PLYAERAELTQLN-TALEASFAAAATRARGALFETLRDPLLREAVFLSNPSALER-V -- -HWNALLARTSAP--EEAAREYDAYLESARQGL IDFLDDEAVAEALFISNPSALTR-I ---KWNDLLKRLVIG--DVARHEYSTYLERARQGL INFLDDDAVSEALFI SNPSARAR-I ---HWNERLGRLKQR--VEVDQEYAVFLESARQALIDFVNDEDVEQAVFISNPTALTR-L -- -RWNEQLARLAQR--TDVDQEYALYLEDARQALIDFLDDEDVAQALFI SNPAALAR-V ---QLNQLQTTLAKMK - IDLDS DF SKYTEQTRQRL I DFLDQPE ISEALFISNPEACKR - I ---EWHNVNAKIVAQE-TTLRPVFANFNEQGRQQL I DF LSRADVSEA IFISNPDAAQR-I -- -EWHSVNAK I IEQE-T SLRS GF IS F SEQVRQQL I DFLSRADVSEA I F I SNPDAAQR- I : $\quad *$ : $\quad$ : : . :

VPYVHSSLQKRNTNIKRIERQLISYLQRLCTKNETTSFFGPIQYGVLTSEQ----QDIEY KENRLENL--TDAQKKQTCRTLFAYLQRVSTKNDTI GEYGPISYGAFN-------QGKLI DTLAECSPERVDNRMRQRLRLAWSYVQRLCAKNDTTSFFGPIAWGEFAPADAAGGEAASF RELIRDRHSRNDSRKKQKLRLGWSYAQRFCAKNDTSSFFGPLAWGRFDTR-----QTANV QELIKDRHSRSDSRKKQKLRLGWSYAQRFCAKNDT S SFFGPLAWGHFKDQ-----QIANV RELRQERHARTDSRKKQKLRLAWSYAQRFCSKNDT S SF FGPLAWGRFDRT-----QVEHV RELIGERFSRTDSRKKQKLRLAWSYAQRFCSKNDTS SFFGPLAWGRFDRL-----QAENV KSLVEGRAGFNDSRKKQKIRLGWSYAQRFCTKNDTCSFFGPITWGQFLAD-----QDALV NALITERHAPHDSRKKQKIRLGWSYAQRFCTKNDTCSFFGPIAWGRFDDR-----QTVLA NTLVTERHTPYDSRKKQKIRLGWSYAQRFCTKNDTCSFFGPIAWGRFDDQ-----QTMLA

$$
: . .{ }^{\star}:{ }^{\star}{ }^{\star} \ldots{ }^{\star \star}:{ }^{\star} .:^{\star \star}::^{\star}: \text { : }
$$

NFNQKE----TERRAFMPYWS IKVLAAQMKECDVFFPYLNVKLSYQYTKTGDS I HSPILE QTRQ-----ITKRKAYFAYQGLQKLLSVIKKD---------------------LMPQ-A EVDFGEGGWIGERRTYFEHWVI SRVAMAMSEDPVLAATLPTSLSPACALIEGALHAPG-N HLTQDDTAWIKDRHTFFENWVVQRLVEQINQQCPDTDLMPLQLNTGCYLHEQTLFMPI -G QLTQNDTTWLKDRHTFFENWVMQRLVEQ INQQCPNTDCMPLKLNASCYLREQHLFMPI -N RITQHGPGWIRERHTFFESWVVQRLVEQLNRHCVDVQFMPLQLNPGCFLNQDTLHLPV-N RITSGEGSWIKERHTFFESWVIQRLVDQLNKQCPDPHFMPFQLNQGCYLIDTTLHMPV-N EVNHAEGNWLRSRKTFFESWVIQRI IGQLNEQCPDANKVPLMLNTGCVLVDDVLFYPL-E NVKWSMGSWLSQRKTFFESWVIQRLVAQ INAQCPEPMC LPLSVNPGCYLQGDVLHYPL-G NVNRSTGPWLSQRETFFESWVIQRLVAQVNAQCPEPACLPLSVNPGCYLQGDVLHYPL-D

$$
{ }^{\star}::: \quad . \quad: . \quad: \quad: \text {. }
$$


WP 010898205.1 BA $\bar{B} 05753.1$ WP 102649326.1 $W^{-} 110653318.1$ WP 048368865.1 $\mathrm{Tg} \overline{\mathrm{IB}}$

WP 117166554.1 $W^{-} 112718210.1$ WP 095098125.1 CUT̄02180.1

WP_010898205.1 $\mathrm{BA} \overline{\mathrm{B}} 05753.1$

WP 102649326.1 WP 110653318.1 WP 048368865.1 $\mathrm{Tg} \overline{\mathrm{I}} \mathrm{B}$

WP_117166554.1 WP 112718210.1 WP- 095098125.1 CŪ̄o2180.1

WP 010898205.1 $\mathrm{BA} \overline{\mathrm{B}} 05753.1$ WP 102649326.1 WP ${ }^{-} 110653318.1$ WP 048368865.1 $\mathrm{Tg} \overline{\mathrm{I}} \mathrm{B}$

WP 117166554.1 $W^{-} 112718210.1$ WP 095098125.1 CŪ̄o2180.1

WP 010898205.1 BA $\bar{B} 05753.1$ WP 102649326.1 $W^{-} 110653318.1$ $W^{-} 048368865.1$ $\mathrm{Tg} \overline{\mathrm{l}} \mathrm{B}$

WP 117166554.1 WP 112718210.1 WP- 095098125.1 CŪ̄o2180.1

WP 010898205.1 $\mathrm{BA} \overline{\mathrm{B}} 05753.1$

WP 102649326.1 WP 110653318.1 WP 048368865.1 $\mathrm{Tg} \overline{\mathrm{I}} \mathrm{B}$

WP 117166554.1 WP 112718210.1 WP_095098125.1 CUT̄̄02180.1
KMVKFPKPYADI IKHLT---YENQS IEQLAKKL---SQPVAWLAKKLRLLEKKRLILIEI

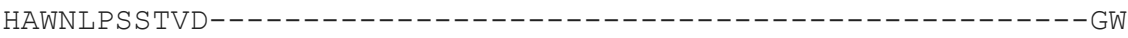
RRIAVDGALRAVLEELEAARAHGIKRDVLRERCVARGLTEQSVDAAIDSVLAKGIASRGV KSQRLNPQTAQVLQY ISEHQGRAATCAGILNNC--PQDAAGTLRDLLEHLVSKRIVRRGW KSQRLTPLTAQVLHT INAQHKEDVTFKQ ILNAC--SDISPYTLRDLLDHLVNKRIVRRGW KRRQVSALTARVLGYIQCQSAVAPTLFGLQAEL--QDVSAGQLRDL I DHLVAQQIVRRGW KRQTLNPLTAQVLDY IQRPASSAPTFYGLLAAL--PQADAGQLEALLEHLVARQ I IRRGW KSRQLSGDMLN IVRLLQ-HHAHVYNCDS LLAKL---MI DNDV---ALKRL I DAG I IKIGF KSRRLTGPTFDVLKALT---MAVVSEKQLRDRL---DNNPGQ---VVKHLISAGIVQRGF KSRRLAGPILEVLKALT---LEVVSEKQLRHRL---NSDPGP---VVEHLIGAGIVQRGF

PLS ITEPDSLNELRSWILKIKDVEHPDLSLWQERLEWLWKVKQAYPYMTL-GQKQELFHK KL-VRER-----------VEDCNDPRSEIWIRI LDQLEETRHLFETSTQPNEKEQALAQ RIAAGLADPLGALRAY-LAHLDAGHPRTRFWRELFESLERERLRFATGGL-DERRAALAD QMS PRERHPIVQLQRC-LANAGVSDPFNQLWQSRLEALEGLRRDYAHGDL-MRRTECLER DIS PRERNPIVRLQHY-LATTGVS PDFQKAWHSRLHALEQARCDYANGDL-IRRTEILEK QIS PRERKPVQVLHTF-LADARLSDDFRTQWHERLSGLEAIRQDYATGDL-AVRIASLDK QVSPRERQPVQVMLSF-LSDSRLSDAFRAQWRERLTELEATRNRYALGDL-SVRMASLEQ VLSPRVENPLKALADK-LVDAKLPTDFTQQWLNTFTELESQREAYASGSL-EQRQIALVN QLSPRDPAALTTILEA-MRTAALPERFVAHWSECFQRLERQRETYAGGDL-QQRQQALAA QISPRDPAALTT ILQA-MRTATLPDSF IAHWSECFRCLEGQRE IYASGDL-HQRQQALVA :

LESTFTEWTGEQPRRLGGE IYADRNLLYEECHGP LNNIKIGGS I KHVLKQDVPKWLS ICA AEKLFTNITGEAFQGENQQYFADKTLLFEEAHDQQ-YLPV--TLSVDLWTELDHALLIHA CETLLAE-NGIDVSREQGKMYVGRFPFYEDCARNV-RVRIGGALRRA IDTELVPLMALYD LNQLLGE-AGVDLSRETGAMYVGRYPLYEDCSRNM-NI S LGQAMLDQVNQELAPLMRINQ LNRLLGE-AGVDLCRESGTMYVGRYP IYEDCSRNI-NISFGQTVFHQVNKELAPLMRINQ LNRLLGE-AGVDISRESGAMYVGRYPVYEDCSRNI-DIS LGGALLDQVNADLAPLMRI HQ MNLLLSE-AGVDLSRESGAMYVGRYPVYEDCSRNI-EVSLGRSLLEQVNADLAPLMRIHQ LNNLLSA-ANVSLSRSSGEMYVGRYPVYEDCSRDT-QVSFNQTIKKHIEEDFTPLILLYQ MNQTLSD-AGVSLARDSGKMYVGRYPVYEDCARAS -TLTFNRKLQQALDVDFAPLMSLYQ MNQALSD-AGISLVRESGKMYVGRYPVYEDCARAS-TLTFSRSMQQALDEDFAPLMSLYQ

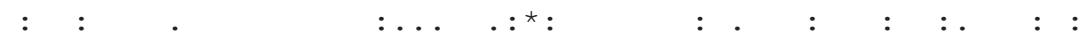

KHGEQRRKQEQALAQEIFKMMY PNEDSVP--FLKFVHDLSNHPDV----------HTWEK KLRLDIWRSWQN IAVQKFEQLANGNVS I P--LMKWVSYWLRYPPSLDNQSATDLLSPW-RLAGAIAAQLSAAYVRI---RAEGDVAVRHDLLSFARELQRHRVA--EEVVATLRPLLRA WLIKAIAHQLNQAF IEVWEQRQVANPGKPVDFLDLLNTLAPLLPALEARI I GDLDQCLET WLIKAIAHQLNSVFVEAWEQRQA INPCRTVDFLDL INTLAPLLPAIETS I IVDLNQRLET WLIKACARQLHGFYAQVWQRLQAEDEHNPVNFLAFLGAVQPVIAHAEAGI IEELDAVLAD WLIKACARQLHAFYVEVWQGFQAADAAAPVDFLAFLGAVQPRLAQVEANI IERTDALLDQ WLTRVTAYELHQHWLGVWSQCCSEYDTDELNILTFLNALKPLQDDIGQQVQTRITTVLQQ WLTRATGVLLHQAWLDVYQLIP PCPDGQDVSLLAFLHLLHPQQAAI QQQVCDRVRTMLNE WLTRATGVLLHQAWLD IFQLIPSRKDGQA I S LLAF LHRLHPQQAT IQQQVCERIRGMLNE - $\quad:$ :

RWQS IKTEIEEA-----------ITHEVASNPKSVVHLSL-DYQDFDRDMAWLTSPDLMI -----LVDRNGY----------LS I KLPDRVIRSWRKE INSFR-------WLLS P DLMV AWSSVVADKQSI------DGEITLDTSDLVRLVSAFEQAGLRASGPQPLGTRVHSPDFLI AWAQLLQDFPGH-------AEVRLCAADVERLISLLNTRL-DVEGFEVFGSDFHSPDILL AWTQLLREFPAQ------SEVRLCATDIDHLISRLNKDF-DVSDFTVFGSDYHSPDLLL AWQQVLSDKHDP-------EQVQLTHEDIERLIVELNTRL-DVRTFSVFGSHFHSPDFLI AWQQVLEGRAEE------AQVHLSAADVERLIEMLNARL-DVQAFTVFGSDFHS PDFLI SWGEILTTVDAKNENAVDSAEIQLTSDDFTVLMSYLNESCPDAQHFEVFGDAFHSPDFML AWQPLLSVVHTE--------ELQLSAAQLEQVLAALNQQCPAAADF PVFGDDFHSPDFML AWQPLLSVVRTE--------EVQLSAEQLAQVLAALHQQC PAAADF PVFGENFHS P DFML

$$
\text { : } \quad \star \star \star:: \text { : }
$$


WP 010898205.1 BA $\bar{B} 05753.1$ WP 102649326.1 $W^{-} 110653318.1$ WP 048368865.1 $\mathrm{Tg} \overline{\mathrm{IB}}$

WP 117166554.1 $W^{-} 112718210.1$ WP 095098125.1 CŪ̄02180.1

WP_010898205.1 $\mathrm{BA} \overline{\mathrm{B}} 05753.1$

WP 102649326.1 WP 110653318.1 WP 048368865.1 $\mathrm{Tg} \overline{\mathrm{I}} \mathrm{B}$

WP 117166554.1 WP 112718210.1 WP- 095098125.1 CŪ̄o2180.1

WP 010898205.1 $\mathrm{BA} \overline{\mathrm{B}} 05753.1$ WP 102649326.1 WP ${ }^{-} 110653318.1$ WP 048368865.1 $\mathrm{Tg} \overline{\mathrm{I}} \mathrm{B}$

WP 117166554.1 WP 112718210.1 WP 095098125.1 CŪ̄o2180.1

WP 010898205.1 BA $\bar{B} 05753.1$ WP 102649326.1 $W^{-} 110653318.1$ WP 048368865.1 $\mathrm{Tg} \overline{\mathrm{l}} \mathrm{B}$

WP 117166554.1 WP 112718210.1 WP- 095098125.1 CUW̄02180.1

WP 010898205.1 $\mathrm{BA} \overline{\mathrm{B}} 05753.1$

WP 102649326.1 WP 110653318.1 WP 048368865.1 $\mathrm{Tg} \overline{\mathrm{I}} \mathrm{B}$

WP 117166554.1 WP 112718210.1 WP_095098125.1 CŪ̄o2180.1
AKKDDDS-----YQVI LGE I HDT IMVW--GWALQFHPEKDRVNEQLVKKIQKSTQHLRML -KGKFETNRFNVAS IVIGELHHGFTA--DGWMFEFHPDKKEINRIV---------- RQA AAGSVDAIREGRFDLVIGEVHPTVHTVSQPVAQP FCPYQDSVRDEVSDTLGNS-- - RMV SSASVEAFNRGDYQ I IVGEVHPAVHTLSQPVAAP FGPFNTQINQQVEAIFQRP----RLV SSAS IEAFNLGHYE I IVGEVHPAVHTLSQPVADP FGPYNSQICQQVETIFQRP----RLI SSTSTDALNQGDYS I I LGEVHPGVHTLSQPVAEP FGPFNKEIEQEVRQIFDGP----RLV ASS STQALNQGDYE I I LGEVHPGVHTLSQPVAEPFGPFNHDINRDVQRLFGGS----RMV SASSQEALNAGDYHLI IGEVHPAVHTLSQPVAAHFS PFNQQINAEVKS LFSSP----RLI AADNLEALNRGEYQVVLGETHPGVHTLSQPVAAP FCPVTAEIDQGVNALLGRE----RLI AADDLEALNRGEYLVVLGETHPGVHTLSQPVAAPFCPFTAEIEQEVNALLGRE----RLV

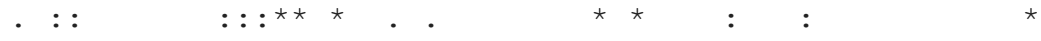

NMLS SKRFKIVPFEYPGT--------TIQMNSFSNSPNEKIPLSQLKVTYTKEGLALTLP MPEKNPSFTWAN--WIFQRKMKSTPQEYPGVSVKLTGHSEYPDEKSFSLYDLEVRRLGDK AADSDSTYQRSHIDWLDVPELWQV--EMPGASARVPGERRVPAARLRLIEREGTLFVEDR LADS PESYQRS H IDWPLQP SYLQL--VLPSGGGCVAAHQQFAAGRAKVLRMNGRLQVVDA LADS PDSYQRS H IDWPLQP FYLQL--VLPSGGGCVEPHQQFAAGRAKVLFVNGRLQVVDT LADS PDSYQRSH IDWPLLECYQQL--I LPSGGGCVPADQRFAVGRARLVMNEGRLRVEDI LADSPDSYQRSH IDWPLLSCYQQL--VLPSGGGCVDPGQRFAAGRARLVMLQGRLHVEDV LADTPTSYQRS H IDWP I INNYQQL--I LPTGGGCVALANQFSAGRAQIVLVDGRLRVTDR LADS PESYQRS H IDWP LVS HYVQL--I LPGGGGSVAAEKRYPAGRARLHCKTGRLTVEDM LADS PESYQRS HIDWPLVPHYAQL--LLPGCGGSVAAEKHYPVGRARLHCKAGRLTVEDM

D------HEEVFYTYN-----GELNTMVHSFFSLPRAVSFS IRTGESTPRLMLGDLVVQR VAVFLQGTDNALMFYP PAYGFNETSFF PFALFCS PMME P S SNYSKGRHKAIKLGNVTLIR ASGL---VEHLLTVLP-----GDFHRAAFAL-------AADVLGAGIAERIRLGHTILKR LGQF---SEDLLCVYS-----TPLHRLGFAL-------AGSAVAKHDHRRIWLGRTLYKR LGQF---SEDLLCVYP-----TPLHRIGFTL-------ADSAVAKHEYRRIWLGRTLYKR AGQF---SEDLVCVYP-----TPMHRLGFAL-------AGSVVAKNDRRRIGLGKTLYKR AGEF---AEDLLCVYS-----TPMHRLGFAL-------AGSAVARGDRRRIWLGKTLYKR LGYF---NEDLLCVHN-----TQLHQLLFDL-------AGDI I PRHECRR I LFNRS IYKR DGAF---HEDLLCVSG-----TSLHQLLFRL-------AGDVLPRHEPRRIRVNRTLYKR DGAF---HEDLLCVNS-----TALHQLLFQL-------AGDMLPRHEPRRIRVNRTLYKR

AKWH ISKQDVWSKKHTGSS IELFYEAYRFHLKESLPKEGFAKLPGQPKPVYVNFTSYFLI EHWVFEPTDWGEVHASLDGFQQMACLQE IKVKYQLPQVGYLRFSQELKPVWFDFNNPFCV RTWTLQASALP IAERVGESMPAF LAWRRWADALGLPRH I FVKADTEPKPVFVDF DCPLSL ASWLFARDRLPEPKGS IDELEHTLQWRAWAGAHGL PRYAFVKIDTEPKPLFLDFDNPLSF ASWLFTREQLPEPKGTIEELEYTMQWRAWAVSQGLPRYAFIKIDTEPKPLFLDFDNPLSL ASWWFS PEQLPCSEFSVDKLDDVLAWRAWAVEHGLPRYVFAKIDIEPKPIFIDFDNPLSL ASWWFTQEQFPCPEFSVDKLDDTLAWRAWAQEHGLPRYVFAKIDIEPKPI F I DFDNPLSF RTWSFESSDWPVS--SKDELGLFVQWRLWKDKYQMPRWIFVKCDSEPKPFFVDFDNPLSL RTWAFNAGGWPEA--VSDE FMAF I QWRDWQQRQALPRWVF IKCDSEPKPLF I DFDNPLSL RTWAFNTGGWPEA--VSDEFMAF I QWQEWQQRQALPRWVF IKCDSEPKPLF IDFDNPLSL

$$
\star \quad: \quad . \quad: \quad{ }^{\star} . \quad: . \quad:{ }^{\star} . .:^{\star} \quad: \text {. }
$$

EMLEGLWPDGENCTFSEMLPNLSELWLSKEDQKTHTAE IRLSY---FVERS-------DLFFNMAKKAEWFTFSRMDPGVEDLFL-KDDRGHYCCELRTFA---YRQEITSKDMLMGDALTSVSARAQTLGISEMLPAPHQVWL-ADERGAFCAEFRTS IAGMGDDEGAQSHSPQQH DGISNALKNAGHVKFSEMRPCPDELWL-EEARGRFCCEIRTTF---STCEALAT-----DGI INALKSAGHVKFSEMRPGPDELWL-EDARGRFCCEIRTTF---S INKALAT-----DGVSNSMKKAGHVKFSEMCPAPDQLWL-EETRGHFCCEIRTTF---RDNGVTRDE----DGVSNSMKKATHVKFSEMCPSPDELWL-EEARGRFCCEIRTTF---TSDGVVPDEQ---DALVSALKKAKVIRVSEMLPDPNALWF-ADKRGKFCAEIRTSMI IS DNNKPVSSEQYE-DALATALKKARVIQVSEMLPTPDELWF-NDARGRVCCEIRTTF---SPIKQETTENAE-DTLATALKKARVIHVSEMLPTPDELWL-NDTRGRVCCEIRTTF---SP IKQEASENAE-: . . * * $\quad::: \quad:$. . ${ }^{\star}:{ }^{\star}$ 


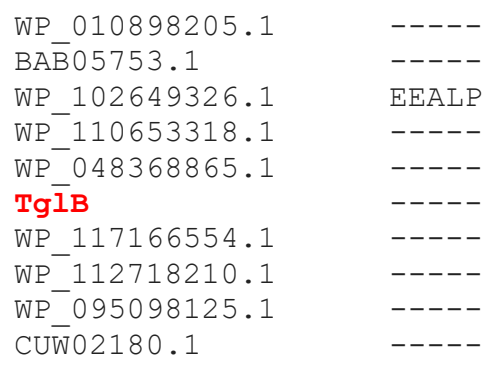

Figure S6. Sequence alignment of TglB homologs. The protein sequence for TglB was used to identify homologs via BLAST search. Homologs with E-values < 1E-39 were aligned by sequence with MUSCLE (MUltiple Sequence Comparison by Log- Expectation), ${ }^{1}$ and highly conserved residues were targeted for alanine replacement. Shown in red are residues replaced with alanine for enzymatic activity assessment in this study. 


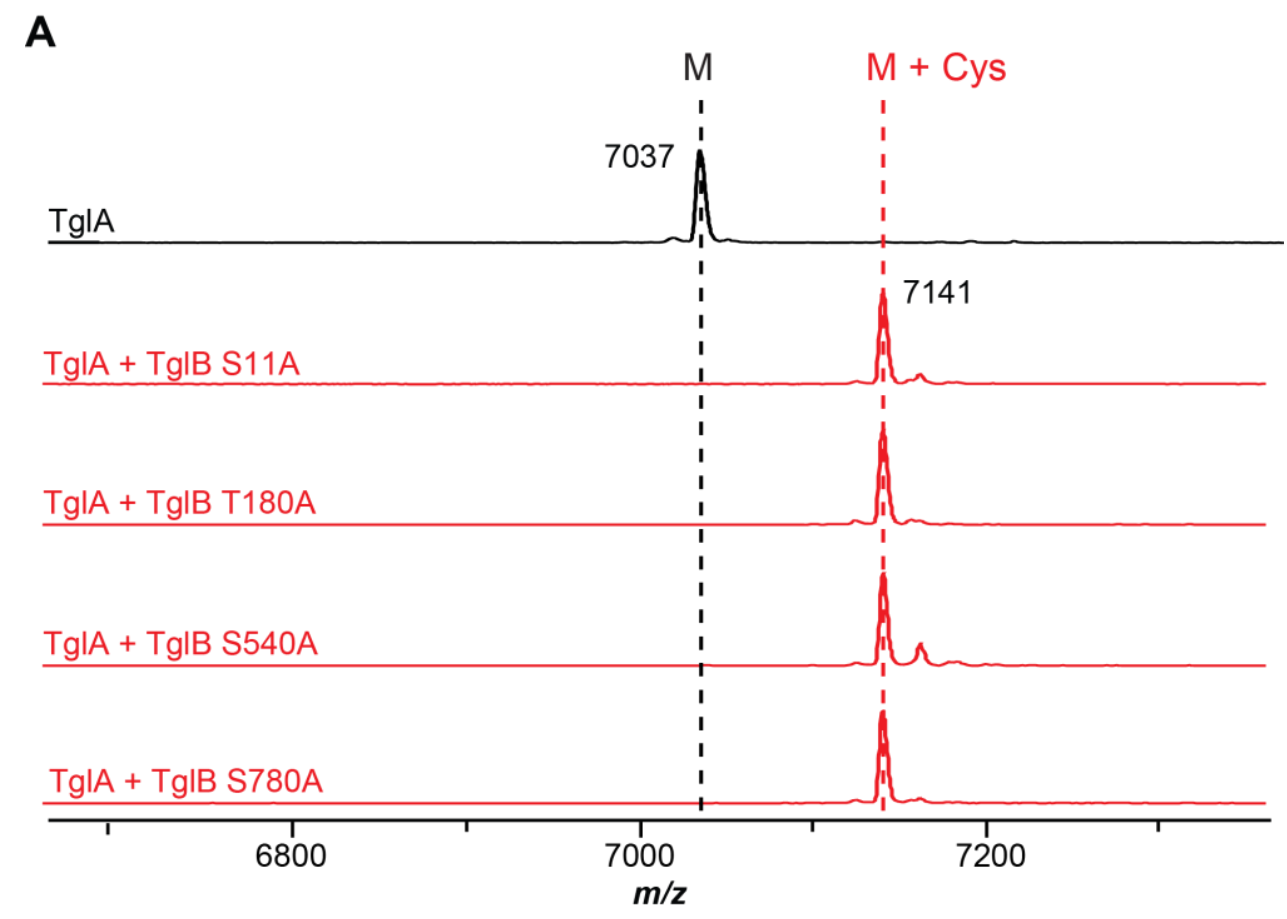

B

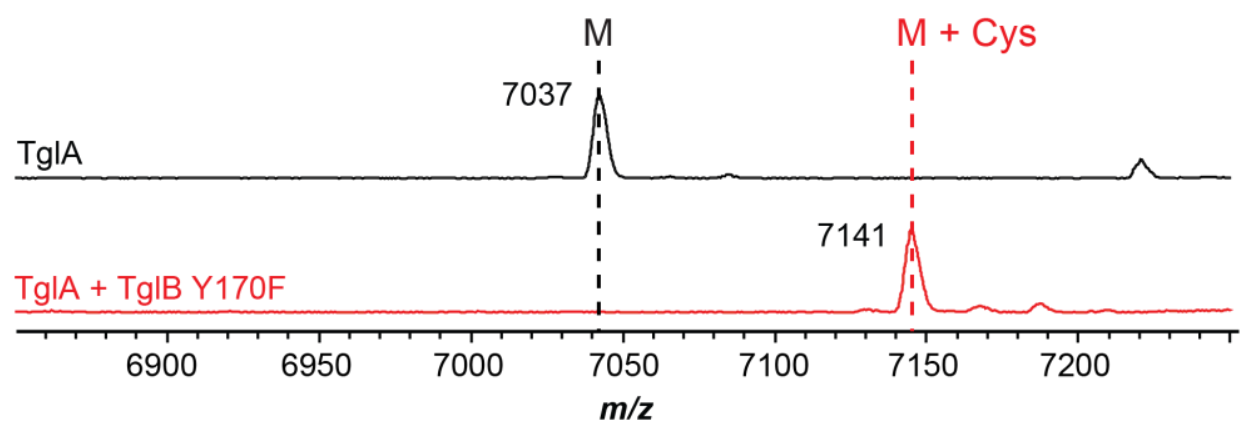

Figure S7. Mutagenesis of selective Ser, Thr and Tyr residues of TglB does not support the ping-pong mechanism in Figure S5. MALDI-TOF-MS spectra of TglA co-expressed in E. coli with TglB variants in which selective (A) Ser or Thr residues were replaced with Ala, (B) a Tyr residue was replaced with a Phe. All experiments were done under the standard TglB assay conditions. 


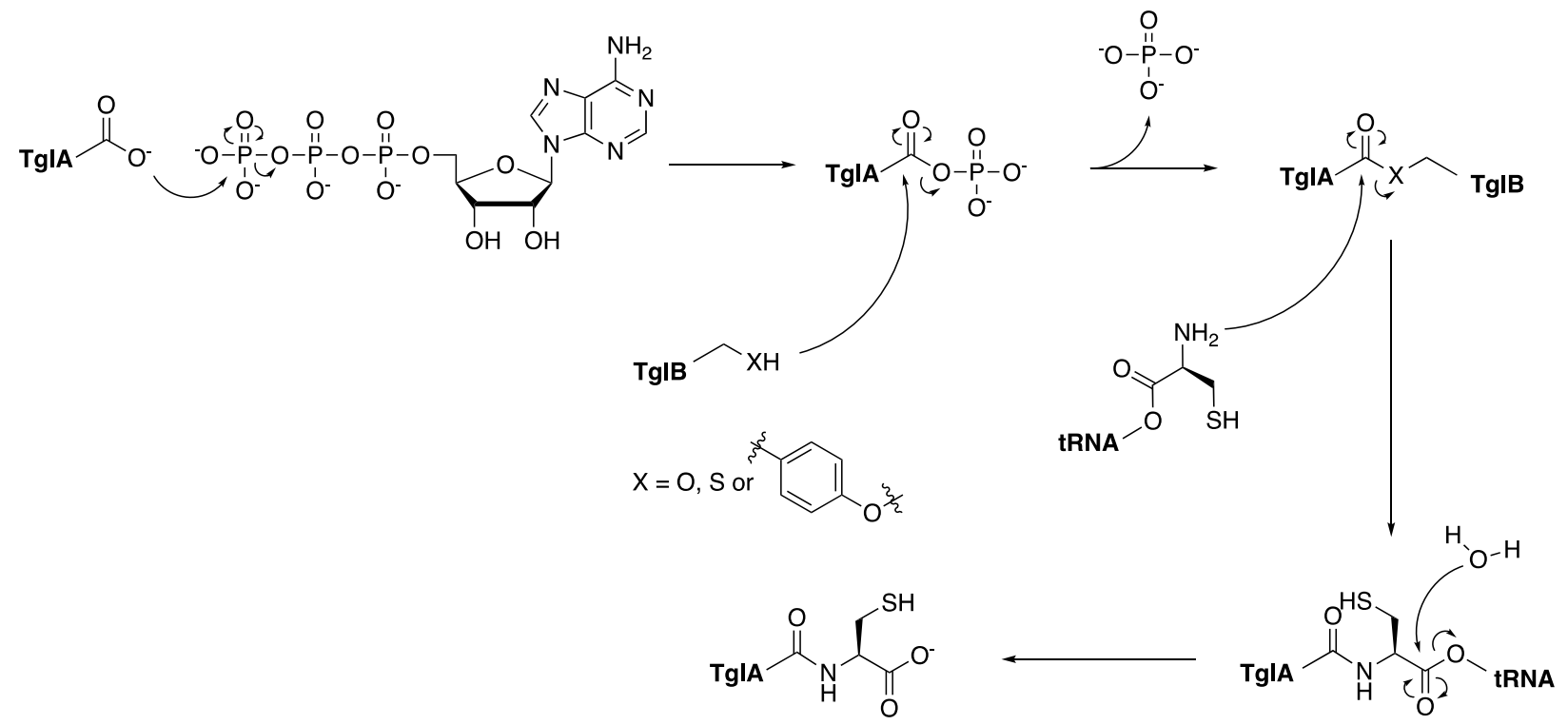

Figure S8. A potential mechanism in which TglA is covalently linked to a hydroxyl group on TglB. In this mechanism, the C-terminal carboxylate is first activated by ATP through phosphorylation. Then, a hydroxyl group from a side chain of a residue on TglB attacks the activated carboxylate to load the TglA peptide onto the protein via an ester that in turn can be attacked by the amino group of the cysteinyl-tRNA. Subsequent hydrolysis of the tRNA would produce the final product. 

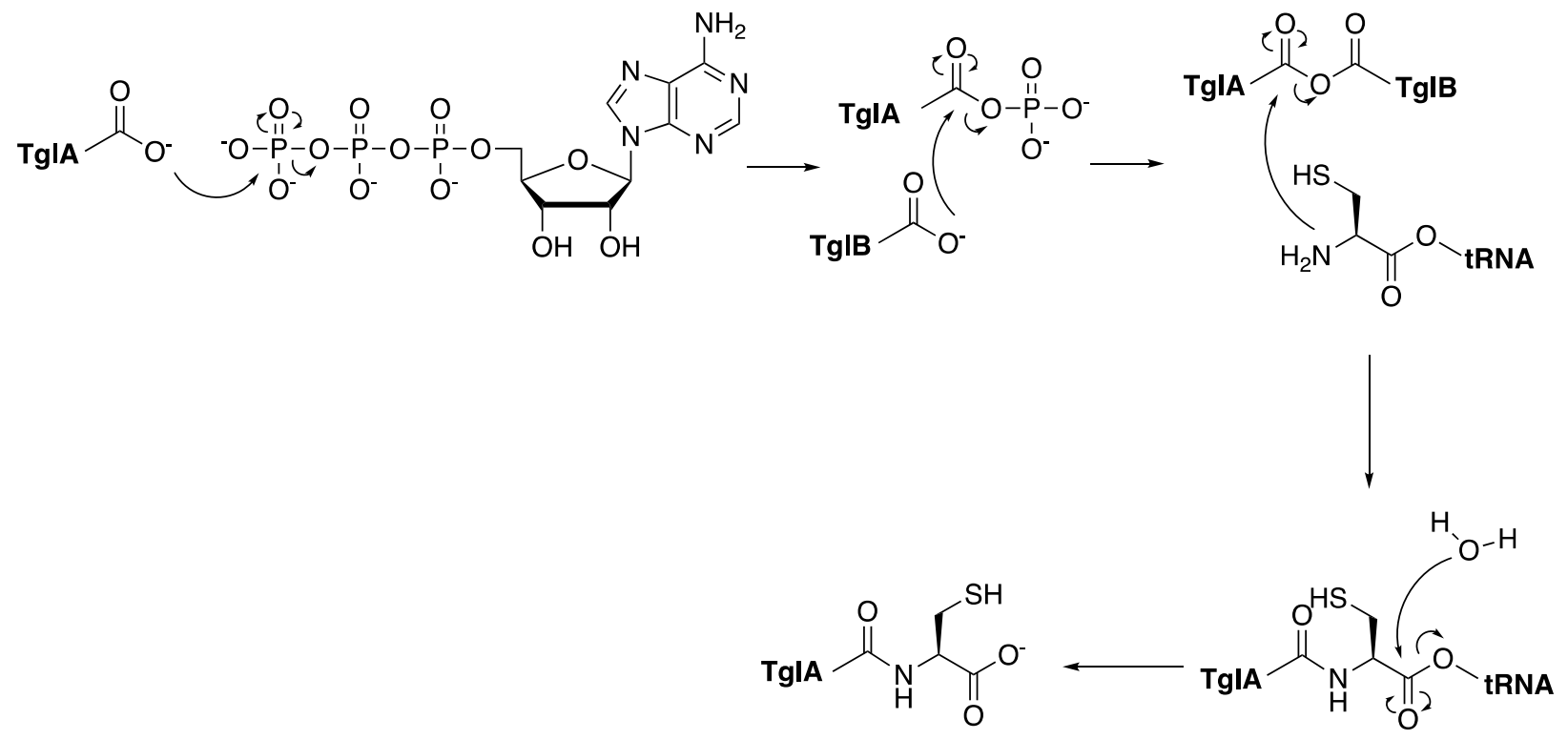

Figure S9. A potential mechanism in which the C-terminus of TglA is first activated by ATP, followed by attack from a carboxylate residue on TglB to form an anhydride. Attempts to trap such an intermediate with hydroxylamine or sodium borohydride were unsuccessful. 

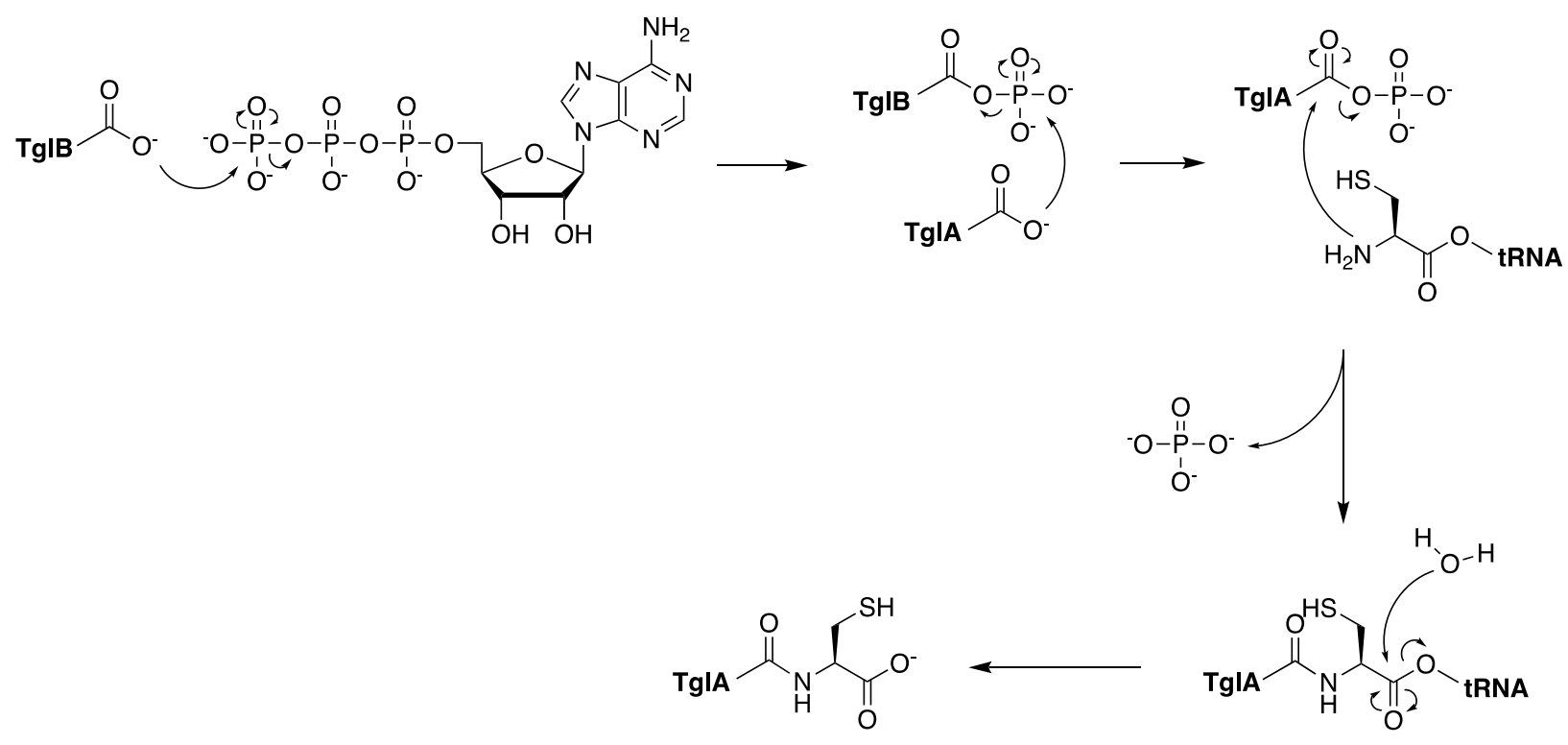

Figure S10. A potential mechanism with a covalent acyl-phosphate intermediate. In this mechanism, a phosphate group is first transferred from ATP onto a carboxylate containing residue on $\mathrm{TglB}$, before further reaction with the TglA peptide. 


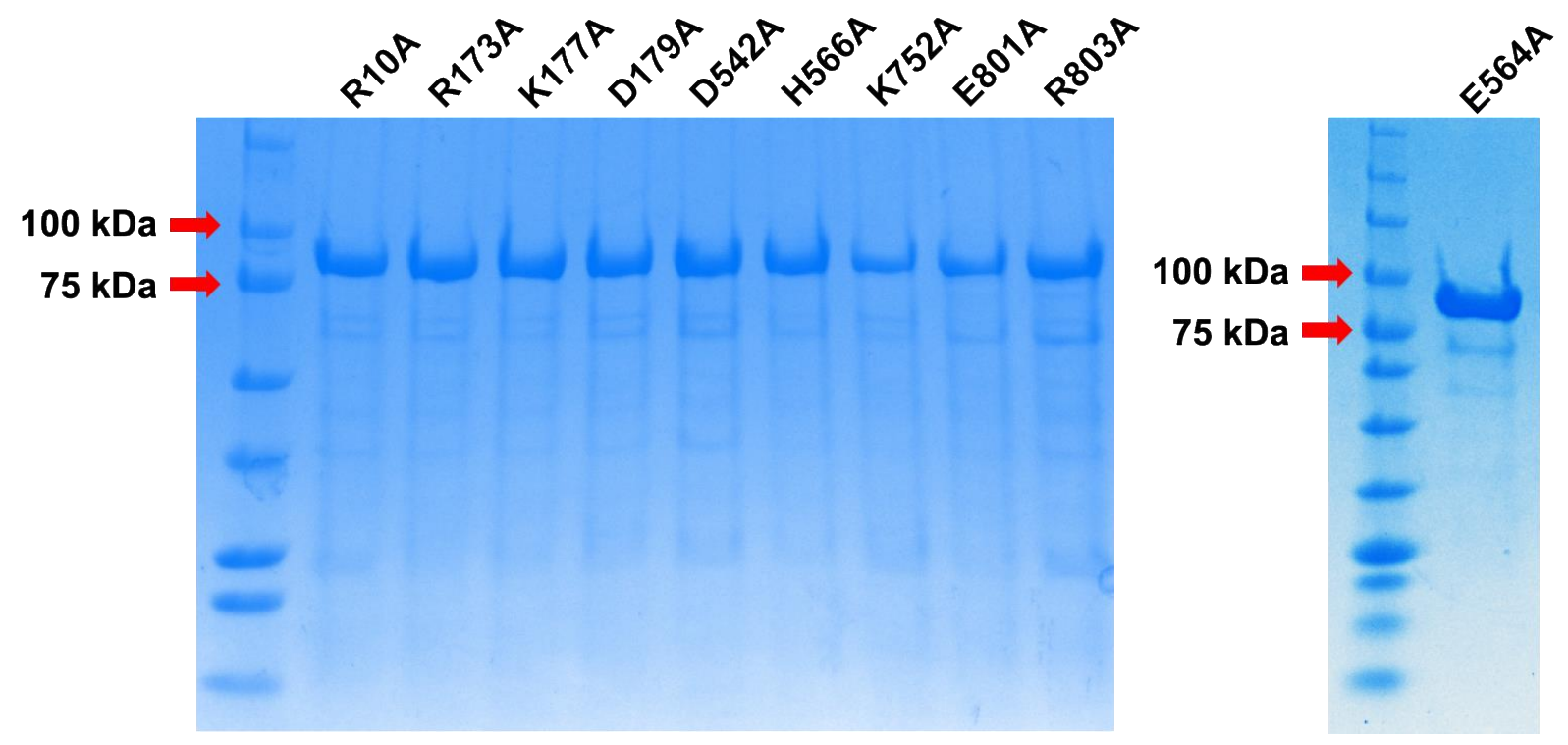

Figure S11. SDS-PAGE analysis of TglB variants with diminished activity. All proteins were expressed as $\mathrm{N}$-terminal His6-tag fusions. 


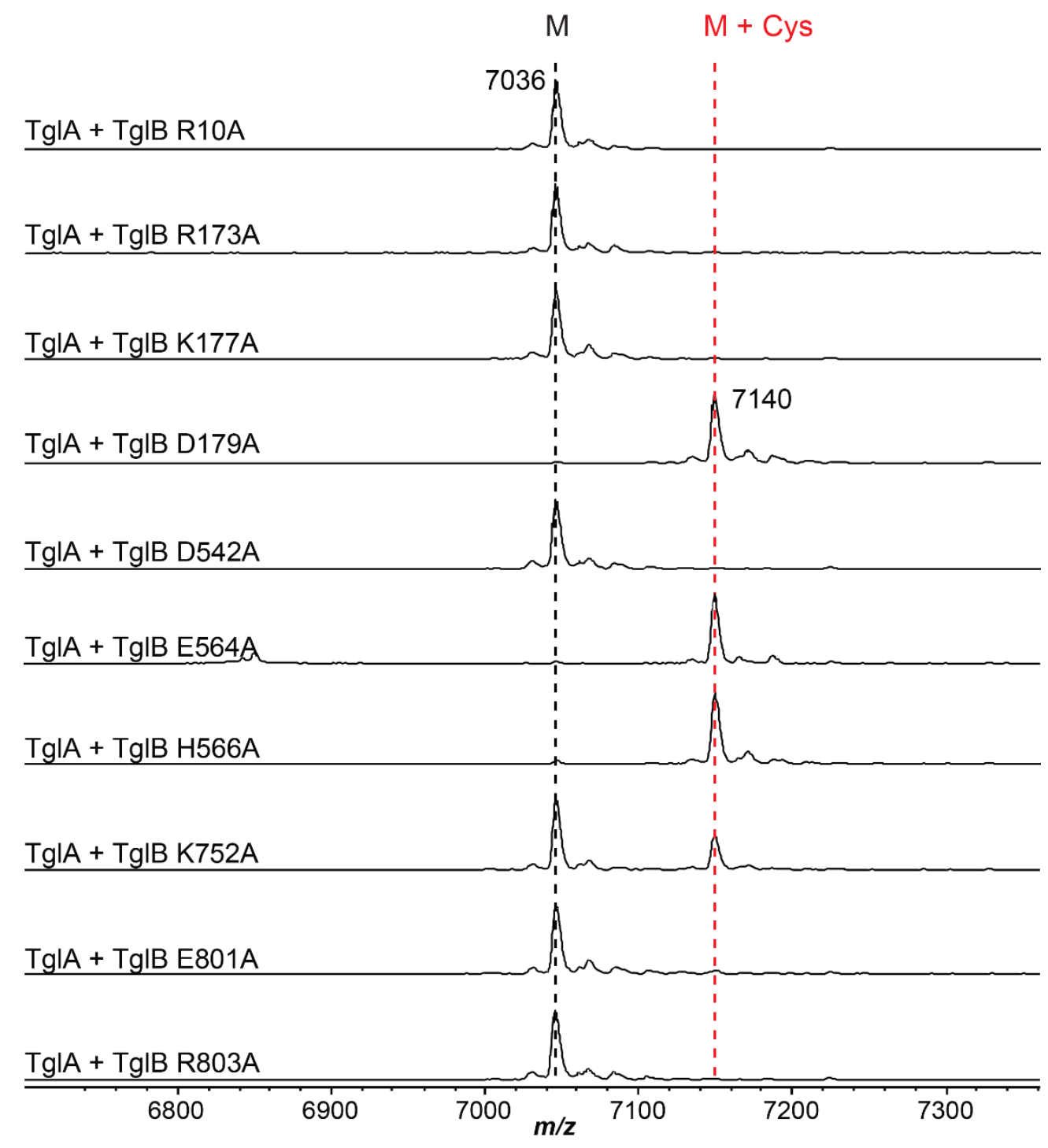

Figure S12. MALDI-TOF-MS spectra of in vitro cysteinylation of TglA with TglB variants. The in vitro activity of each TglB variant mirrors the activity observed when co-expressed with TglA in E. coli (Table S1). All experiments were done under the standard TglB assay conditions. 


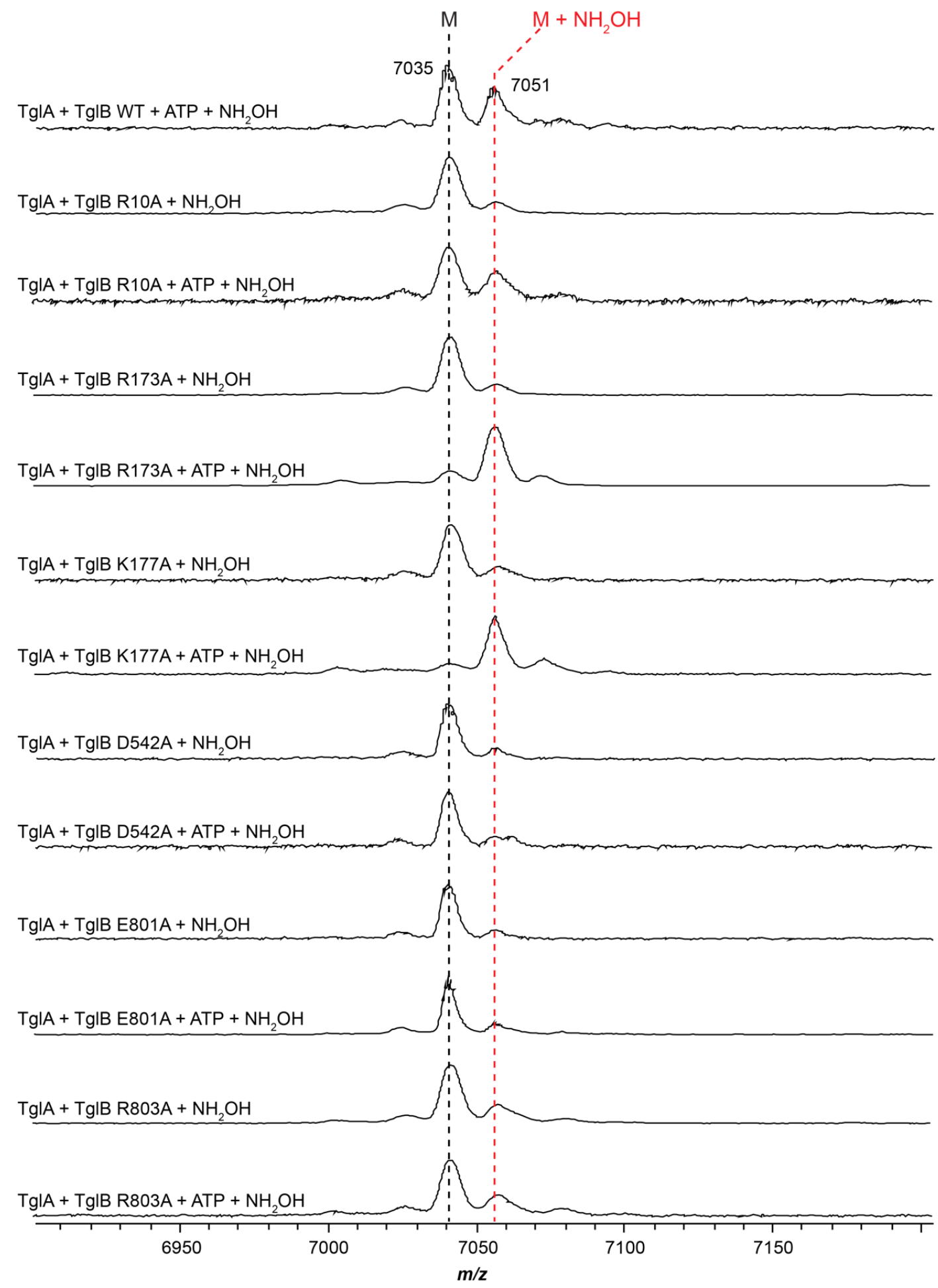

Figure S13. MALDI-TOF-MS spectra of $\mathrm{NH}_{2} \mathrm{OH}$ quenching assays with TglB mutants that do not conjugate Cys to TglA. (TglA, calc. $[\mathrm{M}+\mathrm{H}]^{+}, 7037$; TglA_NHOH, calc. $[\mathrm{M}+\mathrm{H}]^{+}$, 7052). Assays were performed by reacting $50 \mu \mathrm{M}$ TglB and $50 \mu \mathrm{M}$ TglA in the presence or absence of ATP $(5 \mathrm{mM})$ for $5 \mathrm{~min}$ at $30{ }^{\circ} \mathrm{C}$. Then assays were quenched with $\mathrm{NH}_{2} \mathrm{OH}$ to a final concentration of $1 \mathrm{M}$ and incubated at $30{ }^{\circ} \mathrm{C}$ for another $20 \mathrm{~min}$. 
Table S1. Activity of alanine-substituted TglB proteins. Sixteen positions of TglB were targeted for alanine replacement by site-directed mutagenesis using the alignment of Figure S6. These proteins were assayed for enzymatic activity by co-expressing with TglA and analyzed using MALDI-TOF-MS. +++ indicates enzyme activity roughly equal to wild-type (full conversion to product); ++ indicates modestly reduced enzyme activity ( $>50 \%$ conversion to product) ++ indicates severely reduced enzyme activity (low but detectable product formation); - indicates no detectable enzyme activity (no observed product formation).

\begin{tabular}{|c|c|c|c|}
\hline TgIB Protein & $\begin{array}{c}\text { Relative } \\
\text { Product }\end{array}$ & TgIB Protein & $\begin{array}{c}\text { Relative } \\
\text { Product }\end{array}$ \\
\hline Wild-type & +++ & D404A & +++ \\
\hline R10A & - & D542A & - \\
\hline K161A & +++ & E564A & ++ \\
\hline Y170F & +++ & H566A & ++ \\
\hline R173A & - & R704A & +++ \\
\hline K177A & - & W707A & +++ \\
\hline N178A & +++ & K746A & +++ \\
\hline D179A & ++ & K752A & + \\
\hline R397A & +++ & E801A & - \\
\hline E403A & +++ & R803A & - \\
\hline
\end{tabular}


Table S2. Oligonucleotides used in this study. All sequences are provided $5^{\prime}$ to $3^{\prime}$. Lowercase $m$ indicates $2^{\prime} O$-methylation of the following residue; methylation suppresses random addition of bases at the end of the RNA by T7 RNA polymerase.

\begin{tabular}{|c|c|}
\hline Primer & Oligonucleotide Sequence \\
\hline TglA (-Ala)_F & GCAAGGTCTTTTGAAGCTTGCGGCCGCATAATG \\
\hline TglA (-Ala)_R & CGCAAGCTTCAAAAGACCTTGCTCTCGATGACTTCAATGTC \\
\hline P. syringae tRNA ${ }^{\text {Cys }} \mathrm{F}$ & AATTCCTGCAGTAATACGACTCACTATAGGCCGAGTAGCAAAATGGTTATGCAGC \\
\hline P. syringae tRNA ${ }^{\text {Cys }} \mathrm{R}$ & mUmGGAGGCCGAGGTCGGAATCGAACCGGCGTAGGCGGATTTGCAATCCGCTGCATAACC \\
\hline TglB_Seq_623 & CTGGATCAGAGAACG \\
\hline TglB_Seq_1549 & GGCTGATTGTCGAGC \\
\hline TglA_20mer_F & GAGAACCTGTACTTCCAATCCGCGTTGTTTGAAGAGTTTGACC \\
\hline TgIA R & GGATTGGAAGTACAGGTTCTCCGGATCCTGGCTGTG \\
\hline TglB_S11A_F & CTGGCTTCGCGCTACCGGGTTTGCGGTGC \\
\hline TglB_S11A_R & CAAACCCGGTAGCGCGAAGCCAGAAATAGTGTG \\
\hline TglB_T180A_F & CAAAAAATGATGCATCGAGTTTTTTCGGCCC \\
\hline TglB_T180A_R & GAAAAAACTCGATGCATCATTTTTTGAACAGAATCGCTG \\
\hline TglB_S540A_F & CACTTTCACGCTCCGGATTTCCTGATTTCCAG \\
\hline TglB_S540A_R & GAAATCCGGAGCGTGAAAGTGGCTGCCAAATAC \\
\hline TglB_S780A_F & GTCAAGTTTGCTGAGATGTGTCCTGCTCC \\
\hline TglB_S780A_R & CACATCTCAGCAAACTTGACGTGCCCC \\
\hline TglB_R10A_F & CTATTTCTGGCTTGCATCGACCGGGTTTGCG \\
\hline TglB_R10A_R & CGGTCGATGCAAGCCAGAAATAGTGTGAGCTTTCC \\
\hline TglB_K161A_F & CAGCCGCAAGGCTCAGAAACTGCGCCTGG \\
\hline TglB_K161A_R & CAGTTTCTGAGCCTTGCGGCTGTCAGTTC \\
\hline TglB_Y170F_F & CCTGGAGCTTCGCACAGCGATTCTGTTC \\
\hline TglB_Y170F_R & GAATCGCTGTGCGAAGCTCCAGGCCAGG \\
\hline TglB_R173A_F & CTACGCACAGGCATTCTGTTCAAAAAATGATACGTCGAG \\
\hline TglB_R173A_R & GAACAGAATGCCTGTGCGTAGCTCCAG \\
\hline TglB_K177A_F & GATTCTGTTCAGCTAATGATACGTCGAGTTTTTTCGG \\
\hline TglB_K177A_R & CGTATCATTAGCTGAACAGAATCGCTGTGC \\
\hline TglB_D179A_F & GTTCAAAAAATGCTACGTCGAGTTTTTCGGC \\
\hline TglB_D179A_R & CTCGACGTAGCATTTTTTGAACAGAATCGCTGTG \\
\hline TglB_R397A_F & CATGTATGTCGGTGCATACCCGGTCTACGAGGATTG \\
\hline TglB_R397A_R & CGGGTATGCACCGACATACATGGCGC \\
\hline TglB_E403A_F & GGTCTACGCAGATTGTTCGCGCAATATCGATATC \\
\hline TglB_E403A_R & GCGAACAATCTGCGTAGACCGGGTAGCG \\
\hline TglB_D404A_F & GGTCTACGAGGCATGTTCGCGCAATATCGATATCAG \\
\hline TglB_D404A_R & GCGAACATGCCTCGTAGACCGGGTAG \\
\hline TglB_D542A_F & САCTCTCCGGCATTCCTGATTTCCAGTACCTCG \\
\hline
\end{tabular}




\begin{tabular}{|c|c|}
\hline TglB_D542A_R & GAAATCAGGAATGCCGGAGAGTGAAAGTGGC \\
\hline TglB_H566A_F & GAGAGGTCGCTCCGGGCGTGCACAC \\
\hline TglB_H566A_R & CGCCCGGAGCGACCTCTCCCAGAATGATTGAGTAGTCG \\
\hline TglB_R704A_F & CCTTGTACAAAGCTGCCTCGTGGTGGTTCAG \\
\hline TglB_R704A_R & CGAGGCAGCTTTGTACAAGGTCTTGCCCAAG \\
\hline TglB_W707A_F & GCCTCGGCATGGTTCAGTCCAGAGCAAC \\
\hline TglB_W707A_R & GACTGAACCATGCCGAGGCGCGTTTGTAC \\
\hline TglB_K746A_F & GTTCGCCGCTATCGATATCGAACCCAAGCC \\
\hline TglB_K746A_R & CGATATCGATAGCGGCGAACACATAACGGG \\
\hline TglB_K752A_F & CGAACCCGCTCCGATTTTCATTGATTTCGATAACCC \\
\hline TglB_K752A_R & CAATGAAAATCGGAGCGGGTTCGATATCGATCTTGGC \\
\hline TglB_E801A_F & САTTTCTGTTGTGCTATACGTACAACCTTTAGGGACAATGG \\
\hline TglB_E801A_R & GTTGTACGTATAGCACAACAGAAATGCCCGC \\
\hline TglB_R803A_F & CTGTTGTGAAATAGCAACAACCTTTAGGGACAATGGAG \\
\hline TglB_R803A_R & GGTTGTTGCTATTTCACAACAGAAATGCCCG \\
\hline TglA_A50F_F & CAAGGTCTTTTTCTGAAGCTTGCGGCCG \\
\hline TglA_A50F_R & CAAGCTTCAGAAAAAGACCTTGCTCTCGATGACTTC \\
\hline TglA_A50K_F & CAAGGTCTTTAAATGAAGCTTGCGGCCG \\
\hline TglA_A50K_R & CAAGCTTCATTTAAAGACCTTGCTCTCGATGACTTC \\
\hline TglA_A50E_F & CAAGGTCTTTGAATGAAGCTTGCGGCCG \\
\hline TglA_A50E_R & CAAGCTTCATTCAAAGACCTTGCTCTCGATGACTTC \\
\hline TglA_A50Q_F & CAAGGTCTTTCAGTGAAGCTTGCGGCCG \\
\hline TglA_A50Q_R & CAAGCTTCACTGAAAGACCTTGCTCTCGATGACTTC \\
\hline TglA_A50P_F & CAAGGTCTTTCCATGAAGCTTGCGGCCG \\
\hline TglA_A50P_R & CAAGCTTCATGGAAAGACCTTGCTCTCGATGACTTC \\
\hline TglA_A50S_F & CAAGGTCTTTTCTTGAAGCTTGCGGCCG \\
\hline TglA_A50S_R & CAAGCTTCAAGAAAAGACCTTGCTCTCGATGACTTC \\
\hline pTXB1-TglA_F & GGTCTTTGCCTGCATCACGGGAGATG \\
\hline pTXB1-TgIA_R & GTTGGGTTGTCCCATATGTATATCTCCTTCTTAAAGTTAAAC \\
\hline TglA_D40A_F & GACCTGGATGCTATTGAAGTCATCGAGAGCAAG \\
\hline TglA_D40A_R & GACTTCAATAGCATCCAGGTCAAACTCTTCAAAC \\
\hline TglA_I41A_F & CTGGATGACGCAGAAGTCATCGAGAGCAAGG \\
\hline TglA_141A_R & GATGACTTCTGCGTCATCCAGGTCAAACTCTTC \\
\hline TglA_E42A_F & GATGACATTGCTGTCATCGAGAGCAAGGTC \\
\hline TglA_E42A_R & CTCTCGATGACAGCAATGTCATCCAGGTCAAAC \\
\hline TglA_V43A_F & GATGACATTGAAGCTATCGAGAGCAAGGTCTTTG \\
\hline TgIA_V43A_R & GCTCTCGATAGCTTCAATGTCATCCAGGTCAAAC \\
\hline TglA_144A_F & CATTGAAGTCGCTGAGAGCAAGGTCTTTGCCTG \\
\hline
\end{tabular}




\begin{tabular}{|l|l|}
\hline TgIA_144A_R & CTTGCTCTCAGCGACTTCAATGTCATCCAGGTC \\
\hline TgIA_E45A_F & GAAGTCATCGCAAGCAAGGTCTTTGCCTGAAG \\
\hline TglA_E45A_R & GACCTTGCTTGCGATGACTTCAATGTCATCCAGG \\
\hline TglA_S46A_F & CATCGAGGCAAAGGTCTTTGCCTGAAGC \\
\hline TgIA_S46A_R & CAAAGACCTTTGCCTCGATGACTTCAATGTCATCC \\
\hline TglA_K47A_F & CATCGAGAGCGCAGTCTTTGCCTGAAGCTTGC \\
\hline TglA_K47A_R & GCAAAGACTGCGCTCTCGATGACTTCAATGTCATC \\
\hline TglA_V48A_F & GAGAGCAAGGCATTTGCCTGAAGCTTGCG \\
\hline TgIA_V48A_R & CTTCAGGCAAATGCCTTGCTCTCGATGACTTC \\
\hline TgIA_F49A_F & GAGCAAGGTCGCTGCCTGAAGCTTGCGGC \\
\hline TgIA_F49A_R & GCTTCAGGCAGCGACCTTGCTCTCGATGACTTCAATGTCATC \\
\hline
\end{tabular}

(1) Madeira, F.; Park, Y. M.; Lee, J.; Buso, N.; Gur, T.; Madhusoodanan, N.; Basutkar, P.; Tivey, A. R. N.; Potter, S. C.; Finn, R. D.; Lopez, R., Nucleic Acids Res 2019, 47 (W1), W636. 